\title{
EDITORIAL
}

\section{World Oral Health Day: Journey from Oral Health to Mouth Health}

Oral health has been receiving less importance as compared to general health leading to global increase in burden of oral diseases in our society. There are many reasons to explain the cause of this ignorance related to oral health in both rural and urban population which includes lack of public identification of oral health deterioration and awareness about contribution of oral health to overall general health. In addition, oral public health programmers have not been able to achieve the depth and penetration into society required to bring about the change in this societal attitude. Furthermore, mounting evidences of consistent correlation of cause and effect relationship between oral and systemic health, demands prompt action on an individual, family and community level.

In this respect, FDI has started targeted action to control the prevalence of oral disease and make oral health a personal priority for everyone by global celebration of World Oral Health Day (WOHD), which is celebrated on March 20 every year; the day, was first declared by FDI in 2007 and first officially celebrated in 2013. The official theme of this campaign is 'Say Ahh:Act on Mouth Health' for the current year. With this campaign, FDI urges individuals to take tangible action to protect their oral health. Beyond the individual level, the campaign also targeted oral health professionals as key stakeholders to create awareness among patients on the consequences due to alteration in oral health. In addition, assisting them to recognize that preventive care is always the best option, but early detection and treatment are also crucial for ensuring the best outcomes against oral disease like oral precancer and cancer and associated health complications. Furthermore, this type of campaign may draw more attention and commitments from policymakers to address the oral disease burden and also integrate oral health into policies that address other systemic diseases.

Vanitha U Shenoy Editor-in-Chief 